2. L. R. Ford, Jr., Homeomorphism groups and coset spaces, Trans. Amer. Math. Soc. vol. 77 (1954) pp. 490-497.

3. R. H. Bing, Each homogeneous nondegenerate chainable continuum is a pseudoarc, Proc. Amer. Math. Soc. vol. 10 (1959) pp. 345-346.

4. - A homogeneous indecomposable plane continuum, Duke Math. J. vol. 15 (1948) pp. 729-742.

5. - Concerning hereditarily indecomposable continua, Pacific J. Math. vol. 1 (1951) pp. 43-51.

TUlane UNIVERSity AND

St. Mary's Dominican College

\title{
IMMERSIONS OF ALMOST PARALLELIZABLE MANIFOLDS
}

\author{
MORRIS W. HIRSCH ${ }^{1}$
}

The purpose of this note is to prove the following theorem:

$A n$ almost parallelizable $n$-manifold $M$ can be immersed in Euclidean $q$-space $R^{a}$ if $2 q>3 n$.

By immersion $f: M \rightarrow R^{p}$ we mean a continuously differentiable map whose Jacobian matrix has $\operatorname{rank} n=\operatorname{dim} M$ at each point. We denote the differential of an immersion $f$ by $d f$.

A regular homotopy $f_{t}: M \rightarrow R^{n}$ is a homotopy such that each $f_{t}$ is an immersion and $d f_{t}$ is a homotopy of the tangent bundle of $M$ into $R^{n}$. In this case $f_{0}$ and $f_{1}$ have equivalent normal bundles.

We say $M$ is almost parallelizable if the tangent bundle of $M-x$ is trivial, for some $x \in M$.

To prove the theorem, we first observe that if $M$ is not compact, or is bounded, then $M$ is parallelizable, and by $[1,6.3], M$ can be immersed in $R^{n+1} \subset R^{q}$. Hence we assume $M$ is compact and unbounded. Let $B$ be an $n$-ball diffeomorphically embedded in $M$, with bounding $(n-1)$ sphere $S$. Put $M_{0}=M-$ int $B$. By the remark above, there is an immersion $f: M_{0} \rightarrow R^{n+1}$. We consider $f$ as an immersion in $R^{q}$, and we deform $f$ through a regular homotopy near $S$, keeping $f \mid S$ fixed, so that if $X$ is a unit tangent vector to $M$ at point $x \in S$ pointing into $M_{0}$, then $d f(X)$ is the unit vector $e=(0, \cdots, 0,1)$ normal to $R^{q-1}$ in $R^{q}$. We still have $f(S) \subset R^{q-1}$.

Since the immersion $f$ is regularly homotopic to an immersion $M \rightarrow R^{n+1}$, the normal bundle of $f$ is trivial. This enables us to apply a lemma $[2,3.2]$ of M. Kervaire, which implies that the Smale in-

Received by the editors October 10, 1960.

${ }^{1}$ Supported by National Science Foundation Contract NSF G-11594. 
variant of $f \mid S$ vanishes. By [3, E], therefore, there exists an immersion $g: B \rightarrow R^{q-1}$ such that $g|S=f| S$. We consider $g$ as an immersion in $R^{q}$, and we deform $g$ through a regular homotopy, so that if $X$ is the vector above, $d g(-X)=-e$. We now define $h: M \rightarrow R^{a}$ by $h(x)$ $=f(x)$ or $h(x)$, according to whether $x \in M_{0}$ or $x \in B$. It is clear that $h$ is an immersion, and the theorem is proved.

\section{REFERENCES}

1. M. Hirsch, Immersions of manifolds, Trans. Amer. Math. Soc. vol. 93 (1959) pp. $242-276$.

2. M. Kervaire, Sur l'invariant de Smale d'un plongement, Comment. Math. Helv. vol. 34 (1960) pp. 127-139.

3. S. Smale, The classification of immersions of spheres in Euclidean spaces, Ann. of Math. vol. 69 (1959) pp. 327-344.

University of California, Berkeley 